\title{
WITT KERNELS OF BI-QUADRATIC EXTENSIONS IN CHARACTERISTIC 2
}

\author{
HAMZA AHMAD
}

\begin{abstract}
Let $k$ be a field of characteristic 2. The author's previous results (Arch. Math. (1994)) are used to prove the excellence of quadratic extensions of $k$. This in turn is used to determine the Witt kernel of a quadratic extension up to Witt equivalence. An example is given to show that Witt equivalence cannot be strengthened to isometry.
\end{abstract}

\section{INTRODUCTION}

An extension of fields $L / k$ induces a homomorphism $\iota^{*}: W(L) \rightarrow W(k)$ of the Witt groups of (equivalence classes of) quadratic forms. The kernel of $\left(\iota^{*}\right)$ is precisely the (classes of) anisotropic $k$-forms that become hyperbolic over $L$. For fields of characteristic $\neq 2$, the Witt kernels of quadratic and bi-quadratic extensions are known (for example see [5, Theorem 3.2 p. 200] and [4, 2.12 p. 457]). In characteristic 2 , the results for separable quadratic and separable bi-quadratic extensions are analogous to the characteristic $\neq 2$ case. Baeza in $[3,4.3]$ determined the Witt kernel of inseparable quadratic extensions up to Witt equivalence. In $[1,2.8]$, the Witt kernel of such extensions was determined up to isometry. The purpose of this note is to characterise the Witt kernel of bi-quadratic extensions in characteristic 2 (Theorems 10 and 11). As in the characteristic $\neq 2$ case the excellence of quadratic extensions plays an important role. Based on $[1,2.5]$ we establish the excellence of inseparable quadratic extension (Proposition 3 ). In the end we give an example to show that the characterisation (which is up to Witt equivalence) cannot be improved to isometry.

TERminology and Notation. Throughout, the field $k$ will always have characteristic 2. We follow the standard notation as in [2]. In particular, a quadratic $k$-form (or simply a form) $q$ is a map from a finite dimensional $k$-vector space $V$ to $k$ satisfying: (i) For every $a \in k$ and $\mathbf{x} \in V, q(a \mathbf{x})=a^{2} q(\mathbf{x})$, and (ii) $B_{q}(\mathbf{x}, \mathbf{y}):=q(\mathbf{x}+\mathbf{y})-q(\mathbf{x})-q(\mathbf{y})$ is a bilinear map. A form $q$ is called anisotropic if $(q(\mathbf{x})=0$ implies $\mathbf{x}=\mathbf{0})$; and $q$ is called nonsingular if the subspace $V^{\perp}:=\left\{\mathbf{x} \in V \mid B_{q}(\mathbf{x}, \mathbf{y})=0\right.$ for all $\left.\mathbf{y} \in V\right\}=0$. The isometry of forms is denoted by $\cong$, and the orthogonal sum of forms is denoted by $\perp$. The (non-singular) two dimensional quadratic space $(V, q)$ with a basis $\{\mathbf{e}, \mathbf{f}\}$ such that

Received 6th November, 2003

Copyright Clearance Centre, Inc. Serial-fee code: 0004-9727/04 \$A2.00+0.00. 
$q(\mathbf{e})=a, q(\mathbf{f})=b$ and $B_{q}(\mathbf{e}, \mathbf{f})=1$ will be denoted by $[a, b]$. The hyperbolic plane $[0,0]$ will be denoted by $\mathbb{H}$. The orthogonal sum $m \cdot \mathbb{H}:=\mathbb{H} \perp \ldots \perp \mathbb{H}$ ( $m$-summands) is called a hyperbolic form. It is known ([2]) that any non-singular $k$-form $q$ decomposes into

$$
q \cong m \cdot \mathbb{H} \perp\left[a_{1}, b_{1}\right] \perp \ldots \perp\left[a_{r}, b_{r}\right],
$$

with $a_{i}, b_{i} \in k$ such that the form $q^{\prime}=\left[a_{1}, b_{1}\right] \perp \ldots \perp\left[a_{r}, b_{r}\right]$ is anisotropic. The form $q^{\prime}$ and $m$ are uniquely determined by $q$ and are, respectively, called the anisotropic part and the Witt index of $q$. Two forms are Witt equivalent if their anisotropic parts are isometric. The set of Witt equivalence classes of non-singular quadratic $k$-forms with the operation $\perp$ define a group $W(k)$, the Witt group of $k$. Let $L / k$ be a field extension. Then any $k$-form $q$ can also be viewed as an $L$-form (denoted by $q_{L}$ ).

\section{EXCELLENCE OF QUADRATIC EXTENSIONS}

Let $L / k$ be a field extension. An $L$-form $\varphi$ is said to be defined over $k$ if there exists a $k$-form $\gamma$ such that $\varphi \cong \gamma_{L}$. The extension $L / k$ is called excellent if the anisotropic part over $L$ of any non-singular $k$-form is defined over $k$.

It is known that separable quadratic extension are excellent. This is an immediate consequence of the following proposition.

Proposition 1.1. [2, Theorem 4.2 p. 121] Let $k$ be a field of characteristic 2 and let $K=k(\beta) / k$ where $\beta \notin k$ and $\beta^{2}-\beta=b \in k$. If a non-singular anisotropic $k$-form $q$ has Witt index $s$ over $K$, then $q \cong c_{1}[1, b] \perp \ldots \perp c_{s}[1, b] \perp q_{0}$ for some $c_{1}, \ldots, c_{s} \in k$ and a $k$-form $q_{0}$. In particular, if $q$ becomes hyperbolic over $K$, then $q \cong c_{1}[1, b] \perp \ldots \perp c_{r}[1, b]$ for some $c_{1}, \ldots, c_{r} \in k$

Before showing that inseparable quadratic extensions are excellent, we make the following.

REMARK 1.2. Let $K=k(\sqrt{d})$.

(i) By $[1,2.5]$, any non-singular anisotropic $k$-form $q$ can be written as

$$
q \cong q_{1} \perp\left(q_{0} \perp d q_{0}\right) \perp\left(\left[a_{1}, b_{1}\right] \perp d\left[a_{1}, c_{1}\right]\right) \perp \ldots \perp\left(\left[a_{r}, b_{r}\right] \perp d\left[a_{r}, c_{r}\right]\right),
$$

where $q_{0}$ and $q_{1}$ are $k$-forms and $a_{i}, b_{i}, c_{i} \in k,(i=1, \ldots, r)$ such that the Witt index of $q$ over $K=k(\sqrt{d})$ equals $\operatorname{dim}\left(q_{0}\right)+r$. In particular, $q_{1}$ remains anisotropic over $K$, and $\left[a_{i}, b_{i}\right] \perp d\left[a_{i}, c_{i}\right]$ is not hyperbolic over $K$.

(ii) Let $a, b, c \in k$. Then, over $K$, we have

$$
[a, b] \perp d[a, c] \cong[a, b] \perp[a, c] \cong[0, b] \perp[a, b+c] \cong \mathbb{H} \perp[a, b+c]
$$

The first isometry follows because $d \in K^{2}$. To see the second isometry, take $\mathbf{u}_{1}, \mathbf{v}_{1}, \mathbf{u}_{2}, \mathbf{v}_{2}$ to be a basis associated with the presentation $[a, b] \perp[a, c]$, 
and rewrite the form with respect to the new basis $\mathbf{u}_{1}+\mathbf{u}_{2}, \mathbf{v}_{1}, \mathbf{u}_{2}, \mathbf{v}_{2}+\mathbf{v}_{1}$ to get $[0, b] \perp[a, b+c]$.

PROPOSITION 1.3. Let $k$ be a field of characteristic 2. Let $q$ be a non-singular anisotropic $k$-form. Then the anisotropic part of $q$ over $K=k(\sqrt{d})$ is defined over $k$; $i$. e. $K / k$ is excellent.

Proof: Replacing $q$ by its anisotropic part, we may assume that $q$ is anisotropic over $k$. Write

$$
q \cong q_{1} \perp\left(q_{0} \perp d q_{0}\right) \perp\left(\left[a_{1}, b_{1}\right] \perp d\left[a_{1}, c_{1}\right]\right) \perp \ldots \perp\left(\left[a_{\tau}, b_{r}\right] \perp d\left[a_{r}, c_{r}\right]\right),
$$

as in (i) of Remark 1.2. By (ii) of $1.2,\left[a_{i}, b_{i}\right] \perp d\left[a_{i}, c_{i}\right] \cong \mathbb{H} \perp\left[a_{i}, b_{i}+c_{i}\right]$, and therefore

$$
q_{K} \cong\left(\operatorname{dim} q_{0}+r\right) \mathbb{H} \perp q_{1} \perp\left[a_{1}, b_{1}+c_{1}\right] \perp \ldots \perp\left[a_{r}, b_{r}+c_{r}\right] .
$$

Since the Witt index of $q_{K}$ equals $\operatorname{dim} q_{0}+r$ (Remark 1.2.(i)), the anisotropic part of $q$ over $K$ is $q_{1} \perp\left[a_{1}, b_{1}+c_{1}\right] \perp \ldots \perp\left[a_{\tau}, b_{r}+c_{r}\right]$, which is defined over $k$.

As in characteristic $\neq 2$, the excellence property implies (see [3, Lemma 2.1])

COROLlary 1.4. Let $K=k(\sqrt{d})$ be a quadratic extension over $k$. Let $\sigma$ and $\delta$ be non- singular $k$-forms and let $\gamma$ be a non-singular $K$-form. If $\sigma_{K} \cong \delta_{K} \perp \gamma$, then $\gamma$ is defined over $k$.

To determine the Witt kernel of bi-quadratic extensions, one needs a "characteristic 2 " analogue of [3, Proposition 2.11.(a)]. For separable quadratic extensions we have the following statement whose proof is identical to that of [3, Proposition 2.11.(a)].

THEOREM 1.5. Let $k$ be a field of characteristic 2. Let $K / k$ be an excellent extension of $k$. Let $q \cong e_{1}[1, b] \perp \ldots \perp e_{r}[1, b]$ where $b \in k^{*}$ and $e_{1}, \ldots, e_{r} \in K$. If $q$ is defined over $k$, then there exist $c_{1}, \ldots, c_{r} \in k$ such that $q \cong c_{1}[1, b] \perp \ldots \perp c_{r}[1, b]$.

In the case of inseparable quadratic extensions we have

ThEOREM 1.6. Let $K$ be an excellent extension of $k$, and let $d \in k^{*}$. Let $\gamma$ be a non-singular $K$-form such that the form $\gamma \perp d \gamma$ is defined over $k$. Then there exists a $k$-form $\delta$ such that $\gamma \perp d \gamma \cong(\delta \perp d \delta)_{K}$.

The proof will be broken into two lemmas.

LEMMA 1.7. Let $a, b, c, d \in k$ such that the form $\delta \cong[a, b] \perp d[a, b]$ represents $c$. Then $\delta \cong\left[c, b^{\prime}\right] \perp d\left[c, b^{\prime}\right]$ for some $b^{\prime} \in k$.

PROOF: Note that $\delta \cong a \alpha$ where $\alpha$ is the Pfister form $[1, a b] \perp d[1, a b]$. Since $\alpha$ represents $a c$, we have

$$
\delta \cong a \alpha \cong a^{2} c \alpha \cong c \alpha \cong c[1, a b] \perp d c[1, a b]
$$

But $c[1, a b] \cong\left[c, b^{\prime}\right]$ for some $b^{\prime} \in k$. 
Lemma 1.8. Let $K$ be a field extension of $k$, and let $d \in k^{*}$. Let $\gamma$ be a nonsingular $K$-form such that the form $\gamma \perp d \gamma$ represents an element of $k$. Then there exist $a \in k, b \in K$, and a $K$-form $\gamma_{1}$ such that

$$
\gamma \perp d \gamma \cong([a, b] \perp d[a, b]) \perp\left(\gamma_{1} \perp d \gamma_{1}\right)
$$

Proof: Let $(V, \gamma)$ be a non-singular $K$-quadratic space. If $d$ is a square in $K$, then $\gamma \perp d \gamma$ is a hyperbolic form of dimension $2(\operatorname{dim} \gamma)$ which is divisible by 4 . Hence in this case we take $a=b=0$ and $\gamma_{1}$ to be the hyperbolic form of dimension $(\operatorname{dim} \gamma-1)$.

If $\gamma$ is isotropic, then $\gamma \cong[0,0] \perp \gamma_{1}$ and we then let $a=b=0$. So, we may assume that $\gamma$ is anisotropic and $\gamma \perp d \gamma$ represents an element $c \in k^{*}$. So, there exist $\mathbf{v}_{1}, \mathbf{v}_{2} \in V$ not both zero such that

$$
c=\gamma\left(\mathbf{v}_{1}\right)+d \gamma\left(\mathbf{v}_{2}\right) .
$$

If $\mathbf{v}_{1}$ (respectively $\mathbf{v}_{2}$ ) is the zero vector, then $\gamma\left(\mathbf{v}_{1}\right)$ (respectively $\gamma\left(\mathbf{v}_{2}\right)$ ) equals $c$ (respectively $c / d$ ). So, $\gamma$ represents an element $a$ of $k^{*}$ where $a=c$ or $a=c / d$. Therefore $\gamma \cong[a, b] \perp \gamma_{1}$ for some $b \in K$ and a $K$-form $\gamma_{1}$, and the conclusion follows.

Therefore we may assume that $\mathbf{v}_{1}$ and $\mathbf{v}_{2}$ are both non-zero. First assume that $\mathbf{v}_{1}$ and $\mathbf{v}_{2}$ are not orthogonal or $\mathbf{v}_{1}$ and $\mathbf{v}_{2}$ are linearly dependent. Since $\gamma$ is non-singular, in either case the vectors $\mathbf{v}_{1}, \mathbf{v}_{2}$ are contained in a non-singular two dimensional quadratic subspace $V_{0}$ of $V$. Then

$$
(V, \gamma) \cong\left(V_{0},\left.\gamma\right|_{V_{0}}\right) \perp \gamma_{1}
$$

for some $K$-form $\gamma_{1}$. Let $f=\gamma\left(\mathbf{v}_{1}\right)$ and $g=\gamma\left(\mathbf{v}_{2}\right)$. Then by $[1,2.2]$, we have

$$
\left(V_{0},\left.\gamma\right|_{V_{0}}\right) \cong\left[f, f^{\prime}\right] \text { and }\left(V_{0},\left.\gamma\right|_{V_{0}}\right) \cong\left[g, g^{\prime}\right]
$$

for some $f^{\prime}, g^{\prime} \in K$. Since $f+d_{2} g=\gamma\left(\mathbf{v}_{1}\right)+d \gamma\left(\mathbf{v}_{2}\right)=c$, the form $\left[f, f^{\prime}\right] \perp d\left[g, g^{\prime}\right]$ represents $c \in k$. By the previous lemma, there exists $b \in K$ such that

$$
\left[f, f^{\prime}\right] \perp d\left[g, g^{\prime}\right] \cong[c, b] \perp d[c, b]
$$

From the equations (1)-(3) we have

$$
\begin{aligned}
\gamma \perp d \gamma & \cong\left(\left[f, f^{\prime}\right] \perp \gamma_{1}\right) \perp d\left(\left[g, g^{\prime}\right] \perp \gamma_{1}\right) \\
& \cong\left(\left[f, f^{\prime}\right] \perp d\left[g, g^{\prime}\right]\right) \perp\left(\gamma_{1} \perp d \gamma_{1}\right) \\
& \cong([c, b] \perp d[c, b]) \perp\left(\gamma_{1} \perp d \gamma_{1}\right)
\end{aligned}
$$

as desired.

Finally, assume that $\mathbf{v}_{1}$ and $\mathbf{v}_{2}$ are orthogonal and linearly independent. Then by $[1,2.2]$,

$$
\gamma \cong\left[e_{1}, f_{1}\right] \perp\left[e_{2}, f_{2}\right] \perp \gamma_{0}
$$


where $e_{i}=\gamma\left(\mathbf{v}_{i}\right), i=1,2, f_{1}, f_{2} \in K$ and $\gamma_{0}$ is a $K$-form. Since $e_{1}+d e_{2}=\gamma\left(\mathbf{v}_{1}\right)$ $+d \gamma\left(\mathbf{v}_{2}\right)=c$, the form $\left[e_{1}, f_{1}\right] \perp d\left[e_{2}, f_{2}\right]$ represents $c \in k$. By $[1,2.2]$ again, there exists $b, r, s \in K$ such that

$$
\left[e_{1}, f_{1}\right] \perp d\left[e_{2}, f_{2}\right] \cong[c, b] \perp[r, s]
$$

From the equations (4) and (5) we have

$$
\begin{aligned}
\gamma \perp d \gamma & \cong\left(\left[e_{1}, f_{1}\right] \perp\left[e_{2}, f_{2}\right] \perp \gamma_{0}\right) \perp d\left(\left[e_{1}, f_{1}\right] \perp\left[e_{2}, f_{2}\right] \perp \gamma_{0}\right) \\
& \cong\left(\left[e_{1}, f_{1}\right] \perp d\left[e_{2}, f_{2}\right] \perp \gamma_{0}\right) \perp d\left(\left[e_{1}, f_{1}\right] \perp d\left[e_{2}, f_{2}\right] \perp \gamma_{0}\right) \\
& \cong\left([c, b] \perp[r, s] \perp \gamma_{0}\right) \perp d\left([c, b] \perp[r, s] \perp \gamma_{0}\right) \\
& \cong([c, b] \perp d[c, b]) \perp\left(\gamma_{1} \perp d \gamma_{1}\right)
\end{aligned}
$$

where $\gamma_{1}:=\left([r, s] \perp \gamma_{0}\right)$. This completes the proof of the lemma.

REMARK 1.9.

(i) The forms $[a, b], a[1, a b]$ and $a\left[1, a^{2} b^{2}\right]$ are isometric because they are two dimensional forms representing a common element $a$ and have the same Arf invariant (see [6, Lemma 4.4.(i), p. 341]).

(ii) Let $K=k(\sqrt{d}), a \in k$ and $b \in K$. By (i), $[a, b] \cong a\left[1, a^{2} b^{2}\right]$; hence is defined over $k$ because $a^{2} b^{2} \in k$. So in the conclusion of Lemma 1.8, we may assume that both $a$ and $b$ are in $k$.

To complete the proof of Theorem 1.6, note that if $\gamma \perp d \gamma$ is defined over $k$, then it represents an element of $k$. By 1.8 and 1.9

$$
\gamma \perp d \gamma \cong([a, b] \perp d[a, b]) \perp\left(\gamma_{1} \perp d \gamma_{1}\right)
$$

where $a, b \in k$ and $\gamma_{1}$ is a $K$-form. If $\operatorname{dim}(\gamma)=2$, then we are done. If $\operatorname{dim}(\gamma)>2$, then corollary 4 implies that $\gamma_{1} \perp d_{2} \gamma_{1}$ is defined over $k$. The assertion of the theorem follows by induction.

\section{WITT KERNELS OF BI-QUADRATIC EXTENSIONS}

We start with the inseparable case first. One distinguishes between two types of inseparable bi-quadratic extensions: the purely inseparable case where $L=k\left(\sqrt{d_{1}}, \sqrt{d_{2}}\right)$ with $d_{1}, d_{2} \in k$; and the case $L / k$ contains an intermediate separable extension. In the latter case, $L=k(\beta, \sqrt{d})$ for some non-square element $d \in k$ and $\beta \notin k$ such that $\beta^{2}-\beta=b \in k$.

THEOREM 2.1. Let $L / k$ be an inseparable bi-quadratic extension over $k$. Let $q$ be an anisotropic non-singular $k$-form such that $q$ is hyperbolic over $L$. 
(i) If $L=k\left(\sqrt{d_{1}}, \sqrt{d_{2}}\right)$ with $d_{1}, d_{2} \in k$, then $q$ is Witt equivalent to a form of the shape

$$
\left(q_{1} \perp d_{1} q_{1}\right) \perp\left(q_{2} \perp d_{2} q_{2}\right)
$$

for some $k$-forms $q_{1}$ and $q_{2}$.

(ii) If $L=k(\beta, \sqrt{d})$ where $d \in k-k^{2}, \beta \notin k$ and $\beta^{2}-\beta=b \in k$, then $q$ is Witt equivalent to a form of the shape

$$
\left(c_{1}[1, b] \perp \ldots \perp c_{r}[1, b]\right) \perp\left(q_{0} \perp d q_{0}\right)
$$

for some $c_{i} \in k(i=1, \ldots, r)$ and a $k$-form $q_{0}$.

Proof: For (i), let $K=k\left(\sqrt{d_{1}}\right)$. If $q$ is hyperbolic over $K$, the theorem follows immediately from $[1,2.8]$. So, assume $q_{K}$ is not hyperbolic. Let $\varphi$ denote the anisotropic part of $q$ over $K$. By proposition, $\varphi$ is defined over $k$. Since $q$ is hyperbolic over $L=K\left(\sqrt{d_{2}}\right), \varphi_{L}$ is hyperbolic; hence there exists a $K$ - form $q_{2}$ such that $\varphi \cong q_{2} \perp d_{2} q_{2}$. By Theorem 1.6, we may assume that $q_{2}$ is a $k$-form. Consider the $k$-form $\alpha:=q$ $\perp-\left(q_{2} \perp d_{2} q_{2}\right)$. Over $K$, the form $\alpha$ is hyperbolic because (in $\left.W(K)\right)\left[\alpha_{K}\right]=\left[q \perp-\left(q_{2}\right.\right.$ $\left.\left.\perp d_{2} q_{2}\right)_{K}\right]=[\varphi \perp-\varphi]=0$. So, by $[1,2.8], \alpha$ is Witt equivalent (over $k$ ) to $q_{1} \perp d_{1} q_{1}$ for some $k$-form $q_{1}$. Therefore in the Witt ring of $k$ we have

$$
\left[q \perp-\left(q_{2} \perp d_{2} q_{2}\right)\right]=\left[q_{1} \perp d_{1} q_{1}\right]
$$

or equivalently,

$$
[q]=\left[\left(q_{2} \perp d_{2} q_{2}\right) \perp\left(q_{1} \perp d_{1} q_{1}\right)\right]
$$

as desired.

For (ii), we let $K=k(\beta)$. If $q_{K}$ is hyperbolic, then we are done by 1.1 . So, assume that $q_{K}$ is not hyperbolic and let $\varphi$ be its anisotropic part. As in part (i), it follows that $\varphi \cong q_{0} \perp d_{2} q_{0}$ for some $k$-form $q_{0}$ and the $k$-form $\alpha:=q \perp q_{0} \perp d_{2} q_{0}$ is hyperbolic over K. Proposition 1.1 implies that $\alpha$ is Witt equivalent (over $k$ ) to $c_{1}[1, b] \perp \ldots \perp c_{r}[1, b]$ for some $c_{i} \in k(i=1, \ldots, r)$. Therefore $q$ is Witt equivalent to $\left(c_{1}[1, b] \perp \ldots \perp c_{r}[1, b]\right)$ $\perp\left(q_{0} \perp d q_{0}\right)$

Using an argument similar to that in the proof of Theorem 2.1 (or similar to [3, Proposition 2.12] together with Theorem 1.5) we get

THEOREM 2.2. Let $L=k(\alpha, \beta)$ be a (separable) bi-quadratic extension over $k$ with $\alpha^{2}-\alpha=a \in k$ and $\beta^{2}-\beta=b \in k$. Let $q$ be an anisotropic non-singular $k$-form. If $q$ is hyperbolic over $L$, then $q$ is Witt equivalent to a form of the shape

$$
\left(e_{1}[1, a] \perp \ldots \perp e_{r}[1, a]\right) \perp\left(f_{1}[1, b] \perp \ldots \perp f_{s}[1, b]\right)
$$

for some $e_{i}, f_{j} \in k(i=1, \ldots, r ; j=1, \ldots, s)$. 
We conclude this note by an example which shows that the Witt equivalence in the conclusions of Theorems 2.1 and 2.2 above cannot be strengthened to isometry.

EXAMPLE. Let $k_{0}$ be a fixed field of characteristic two. Let $k=k_{0}(r, s, t, u)$ where $r, s, t, u$ are algebraically independent elements over $k_{0}$ and set

$$
q \cong[1, r] \perp t[1, s] \perp u[1, r+s]
$$

Let $\alpha, \beta$ (in the algebraic closure of $k$ ) be such that $\alpha^{2}-\alpha=r$ and $\beta^{2}-\beta=r+s$. Then

(i) The form $q$ is anisotropic over $k$ because $r, s, t, u$ are algebraically independent elements over $k_{0}$ (see [5, ex. 1 p. 273]).

(ii) Over the fields $K_{1}=k(\sqrt{t}), K_{2}=k(\sqrt{u}), K_{3}=k(\alpha)$ and $K_{4}=k(\beta)$, the form $q$ is isotropic and have Witt index 1 . We see this as follows: First over $K_{1}, t \in K_{1}^{2}$ and $[1, r] \perp t[1, s] \cong[1, r] \perp[1, s] \cong \mathbb{H} \perp\{1, r+s\}$ (see (ii) of Remark 1.2). Therefore

$$
q_{K_{1}} \cong \mathbb{H} \perp[1, r+s] \perp u[1, r+s] .
$$

Since $r+s$ and $u$ are algebraically independent over $k_{0}(\sqrt{t})$, the form $[1, r+s] \perp u[1, r+s]$ is anisotropic over $K_{1}$, and therefore $q_{K_{1}}$ has Witt index 1 . Similarly, we can show that $q_{K_{2}}$ also has Witt index 1 .

Now over $K_{3}=k(\alpha)$, the form $[1, r]$ is isotropic and $[1, r+s] \cong_{K_{3}}[1, s]$ (for they have the same Arf invariant over $K_{3}$ and represent 1). Therefore, over $K_{3}$,

$$
q_{K_{3}} \cong \mathbb{H} \perp t[1, r+s] \perp u[1, r+s]
$$

and $t[1, s] \perp u[1, s]$ is anisotropic over $K_{3}$ because $s, t$ and $u$ are algebraically independent over $k_{0}(\alpha)$. Therefore, $q_{K_{3}}$ has Witt index 1 . Likewise, $q_{K_{4}}$ has Witt index 1.

(iii) The form $q$ is hyperbolic over the fields $L_{1}=k(\sqrt{t}, \sqrt{u}), L_{2}=k(\sqrt{t}, \beta)$ and $L_{3}=k(\alpha, \beta)$ :

Note that $u \in L_{1}^{2}$ and therefore the form $[1, r+s] \perp u[1, r+s] \cong[1, r+s]$ $\perp[1, r+s] \cong 2 \mathbb{H}$. Since $K_{1} \subset L_{1}$, we have from equation (6) above that

$$
q_{L_{1}} \cong \mathbb{H} \perp[1, r+s] \perp u[1, r+s] \cong 3 \mathbb{H}
$$

That is, $q_{L_{1}}$ is hyperbolic.

Since $\beta$ belongs to $L_{2}$ and $L_{3},[1, r+s] \cong \mathbb{H}$ over $L_{2}$ and $L_{3}$ because $\beta^{2}+\beta+(r+s)=0$. Therefore the form $[1, r+s] \perp u[1, r+s]$ (respectively, $t[1, r+s] \perp u[1, r+s]$ ) is hyperbolic over $L_{2}$ (respectively, $L_{3}$ ). Therefore equation (6) (respectively, equation (7)) implies that $q_{L_{2}}$ (respectively, $q_{L_{3}}$ ) is hyperbolic.

(iv) Theorems 2.1 and 2.2 imply that over $k$ the form $q$ is Witt equivalent to forms of the shape
(a) $\left(q_{1} \perp t q_{1}\right) \perp\left(q_{2} \perp u q_{2}\right)$.
(b) $\left(q_{1} \perp t q_{1}\right) \perp\left(c_{1}[1, r+s] \perp \ldots \perp c_{n}[1, r+s]\right)$. 
(c) $\left(b_{1}[1, r] \perp \ldots \perp b_{m}[1, r]\right) \perp\left(c_{1}[1, r+s] \perp \ldots \perp c_{n}[1, r+s]\right)$.

where $b_{j}, c_{i} \in k$ and $q_{1}$ and $q_{2}$ are non-singular $k$-forms. This Witt equivalence cannot be improved to isometry. For if $q$ is isometric to (a), (b) or (c), then by comparing dimensions we have either $\operatorname{dim} q_{1} \geqslant 2, \operatorname{dim} q_{2} \geqslant 2, m \geqslant 2$ or $n \geqslant 2$. This respectively imply that the Witt index over $K_{1}, K_{2}, K_{3}$ or $K_{4}$ is $\geqslant 2$; contradicting part (ii) of this example.

\section{REFERENCES}

[1] H. Ahmad, 'On quadratic forms over inseparable quadratic extensions', Arch. Math. 63 (1994), 23-29.

[2] R. Baeza, 'Quadratic forms over semilocal rings', Lecture Notes in Mathematics 655 (Springer-Verlag, Berlin, Heidelberg, New York 1978).

[3] R. Baeza, 'Ein teilformensatz für quadratische formen in charakteristik 2', Math. Z. 135 (1974), 175-184.

[4] R. Elman, T.Y. Lam and A. Wadsworth, 'Amenable fields and Pfister extensions', in Conf. Quad. Forms, 1976, (G. Orezech, Editor), Queens Papers on Pure and App. Math. 46, 1977, pp. 445-492.

[5] T.Y. Lam, The algebraic theory of quadratic forms (Benajmin, Cummings Publ. Co., Reading, MA, 1973).

[6] W. Scharlau, Quadratic and hermitian forms (Springer-Verlag, Berlin 1985).

Department of Mathematical Sciences

Saginaw Valley State University

University Center, MI $\mathbf{4 8 7 1 0}$

Cnited States of America

e-mail: hyahmad@svsu.edu 